\title{
iTrack: IoT framework for Smart Food Monitoring System
}

\author{
Amrita Srivastava \\ Department of Computer Science \\ IGDTUW \\ Delhi, India
}

\author{
Ankita Gulati \\ Department of Computer Science \\ IGDTUW \\ Delhi, India
}

\begin{abstract}
In the era of technology advancement, everything requires monitoring and controlling. This paper proposes an IoT framework for facilitating food monitoring for protection of the food, so that it would not get contaminated due to surrounding conditions during storage and transportation. In present scenario, the work done is in terms of the sensed values that have been recorded and a detailed analysis has been performed but automated control alternatives are not present. The proposed solution analyzes temperature, moisture, light as these parameters affect nutritional values of food items and makes the analysis results accessible to the user via a mobile application. A web server is used for storage of data values sensed in real time and also for analysis results. User is alerted via messages along with location of the shipment whenever an emergency occurs.

In this solution, heterogeneous sensors for various domains are employed for sensing the condition of food. The data values with plotting of graphs has been done at remote location so that this data can easily be used for further analysis and the user could be notified if a change in parameters values above a threshold is recorded
\end{abstract}

\section{Keywords}

Real time monitoring; remote logging; nutritive value; mobile application; user alerts

\section{INTRODUCTION}

A food contamination can occur in the production process, but also a large part caused by the inefficient food handling because of inappropriate ambient conditions when the food is being transported and stored [1]. There are many factors leading to food poisoning, typically changes in temperature and humidity are important factors. So the monitoring system capable of measuring temperature and humidity variability during transport and storage is of prime importance [2].

Today almost everybody is getting effected by the food they consume, it's not only about the junk food, but all the packed foods, vegetables, products consumed and used in daily life, as all of them do not offer quality since their temperature, moisture, oxygen content vary from time to time. Majority of consumers only pay attention to the information provided on the packaging, i.e. the amount of ingredients used and their nutritional value but they forget that they are blindly risking their health by ignoring the environmental conditions to which these packets are subjected.

Every product making firm just want to attract more and more costumers towards them their main motive is to sell the product anyhow like by adding more flavors, coloring chemicals and preservatives to increase the taste and appearance but they forget that these money making tactics are actually affecting the consumers health.

\section{PROBLEM STATEMENT}

To ensure food safety it should be monitored at every stage of supply chain. It serves the purpose of preventive consumer health protection by maintaining the required standard ambient conditions needed to preserve the quality of food. The performance and analysis of routine measurements, aimed at detecting changes in the nutritional or health status of the food doesn't guarantee that.

Estimates of food and nutrient intakes defines by the proper standard of values that has been fixed by the food health organization so as to identify potential nutritional inadequacies and inappropriate food consumption patterns. This includes issues specific to the warehouse or cold storage monitoring where it has to be properly monitored at every step.

Food and nutrition monitoring and surveillance involves continuous description of the components of the food and nutrition system for the purposes of planning, policy analysis, program evaluation and trend forecasting[27].

Information collected through monitoring and surveillance must be analyzed and transmitted to decision-makers in an appropriate format and in a timely fashion if it is to be of real value. Dissemination of information must be an interactive process[3],[26].

Thus, Integration of the sensors with remote web server for data logging and a software application which allows distribution of data log as well alert messages is the need of the hour. Real time user notification mechanism is also required to ensure immediate preventive action.

\section{PROPOSED SOLUTION}

A system has been proposed to analyze the ambient conditions under which the food item is being stored and transported. The proposed solution senses the temperature, moisture, light parameters of surrounding environment as these parameters affect nutritional values of food items as shown in Fig1. The values of these parameters are then compared to standard values serving as threshold values for respective parameters. The data values with plotting of graphs has been done at remote location so that this data can easily be used for further analysis and the user could be notified if a change in parameters values above a threshold is recorded. A web server is used for storage of data values sensed in real time and analysis results. User, i.e. storage inventory manager is alerted via messages along with location of the shipment whenever an emergency occurs. An android mobile application is used to facilitate user interaction due to wide penetration of android devices. The aim is to develop a generic platform that can be interfaced with third party applications to enable easy access to all the stakeholders involved in storage and transportation process. 


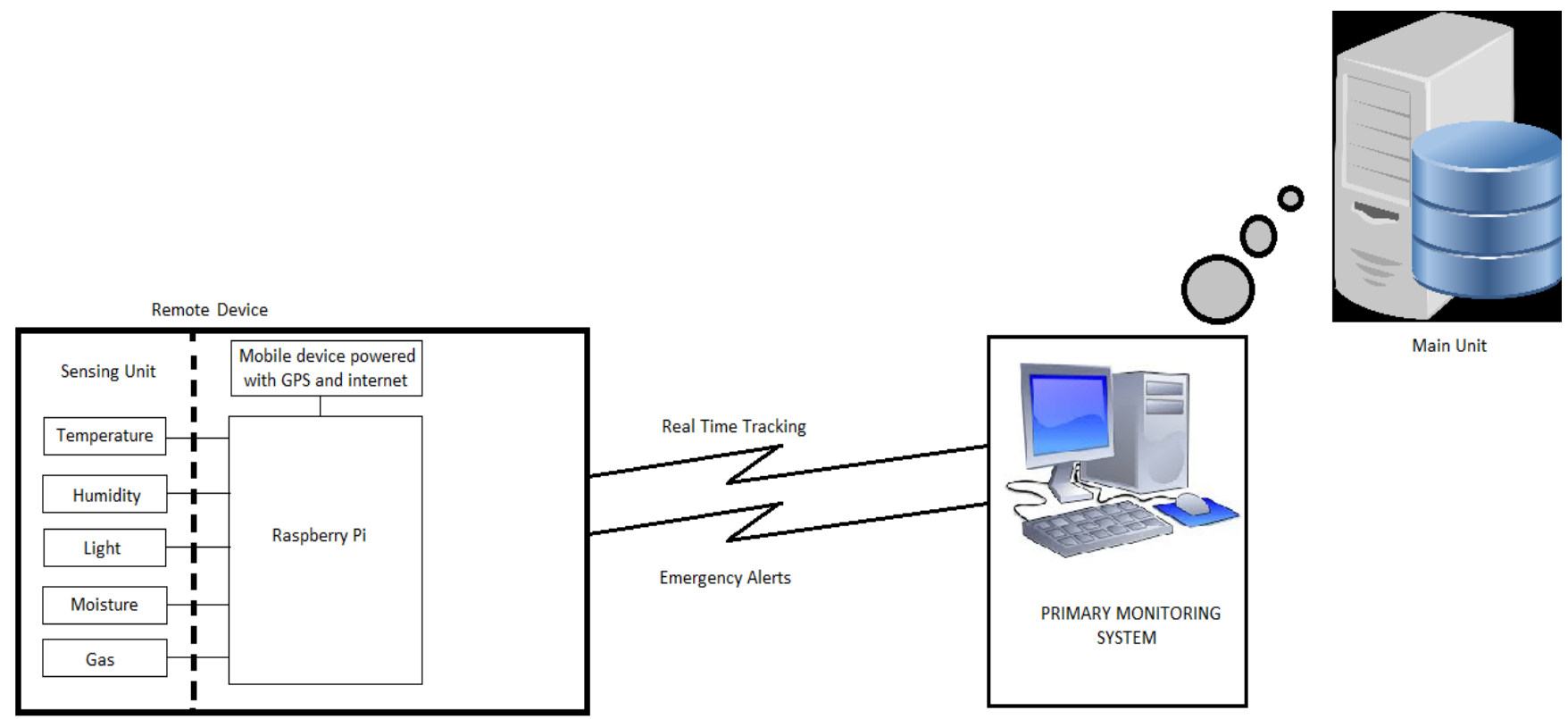

Fig1.Proposed Solution

\section{SYSTEM ARCHITECTURE}

The proposed system consist of three subsystems:

\subsection{Sensors Subsystem}

It basically includes the sensing interface that continuously monitors temperature, humidity, $\mathrm{pH}$ values and light intensity and sends these values to processing subsystem at regular intervals. The particular sensors used for prototype development are listed in Table1.

Today's smart devices peripherals are becoming more integrated and play an important part of our computing experience and also offer the convenience of wireless connectivity.

Selection of a sensor typically depends on following factors:

- Balancing high performance against design complexity and board space

- Balancing ease of use against design complexity and cost

- Balancing high functionality against low-power consumption

- Cost savings and lower space requirements

Table 1. Sensors used for prototype implementation

\begin{tabular}{|l|l|}
\hline Sensors & Specifications \\
\hline Temperature sensor & DHT1 $^{[20]}$ \\
\hline Humidity sensor & DHT11 $^{[20]}$ \\
\hline Light sensor & TSL2561 $^{[21]}$ \\
\hline Magnetic sensor & Hall Effect sensor ${ }^{[22]}$ \\
\hline Ultrasonic sensor & HCSR 04 ${ }^{[23]}$ \\
\hline Air quality sensor & MQ13 \\
\hline
\end{tabular}

\subsection{Processing Subsystem}

The input data from the sensors is analyzed ,i.e. each input value is compared with respective threshold value for each sensor and if an anomaly is recorded an emergency signal is sent to the communication system. Raspberry pi $2^{[18]}$ is selected at processing subsystem as it offers following capabilities:

- Power rating: $800 \mathrm{~mA}(4.0 \mathrm{~W})$

- Memory: $1 \mathrm{~GB}$ (shared with GPU)

- USB ports: 4

- $\quad$ Sizee : $85.60 \mathrm{~mm}$ X $56.5 \mathrm{~mm}$

- Weight: $45 \mathrm{~g}(1.6 \mathrm{oz})$

\subsection{Communication Subsystem}

It provides the user with alert messages upon receiving emergency signal from processing subsystem.

Selection of Communication Module

Bluetooth and WiFi are chosen as communication modules due to their communication ranges and data rates. WiFi is chosen as it offers scalability.

Table 2. Characteristics of communication protocols used

\begin{tabular}{|l|l|l|}
\hline Parameter & $\mathrm{Wi}^{-\mathrm{Fi}^{[25]}}$ & Bluetooth \\
\hline Range & $\sim 100 \mathrm{~m}$ & $10 \mathrm{~m}-30 \mathrm{~m}$ \\
\hline & $2-$ & \\
Data rate & $11 \mathrm{Mbps}$ & $1 \mathrm{Mbps}$ \\
\hline Cost & Higher & Medium \\
\hline Power Consumed & Medium & Low \\
\hline Size & Larger & Smaller \\
\hline
\end{tabular}

\section{SYSTEM METHODOLOGY}

A Graphic user interface(GUI) is designed to assist the user. This GUI is basically for the user monitoring at the server end. Using this application, the user can monitor the sensor values and keep a record of variations. These data values and alert messages can be shared with end user(the one monitoring 
the shipment containing the food items) via Bluetooth or Wi-Fi depending on the proximity of the end user with remote user.

Basically there are three modules by which GUI can be created on Raspberry Pi. The different modules are :

\section{- $\quad$ Tkinter}

- Wxpython

- Jpython

We are using the Tkinter module that supports different GUI to develop on the raspberry pi.

Fig2. shows the front page of GUI which comprises of a label, canvas and two buttons. Hereby we are using only one window which leads to different respective windows whenever the user taps on the buttons present on the second page. This frame is the parent frame that guides you to the two states. When you press the CONTINUE button, there is a command to enter Page Log which is a log page for authentication purpose.

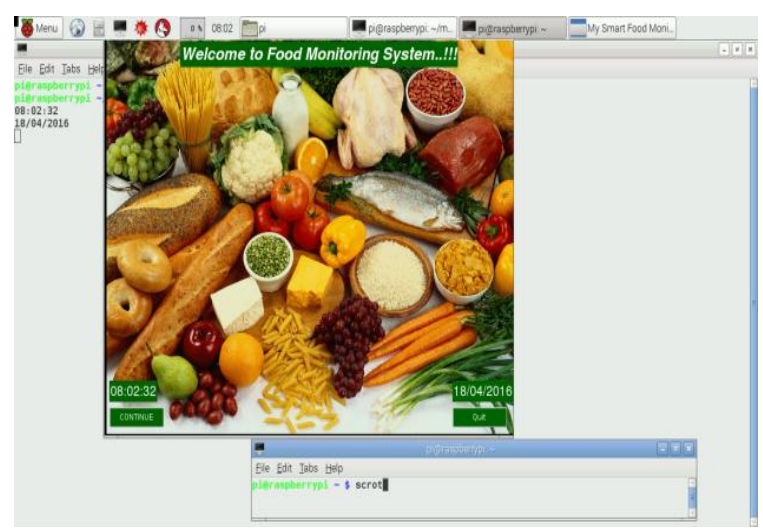

Fig2.MainPage of the GUI

Whenever the user selects the Tracking system, then a new frame named Page Three arises that basically consist of tracking information. If the user wants to see the graph at remote side then the user needs to login on thingspeak.com. After successful login, users can view the graphs showing real time tracking values and variations.

On the Page One, if the user selects sensor data then a frames arises that comprises of labels having names of different sensors. User needs to select the particular sensor and the control will get redirected to show respective values.

A View All button is also provided to provide a comprehensive view of all the sensor values. By tapping this single button, the values of all sensors will appear on the GUI as shown in Fig3..

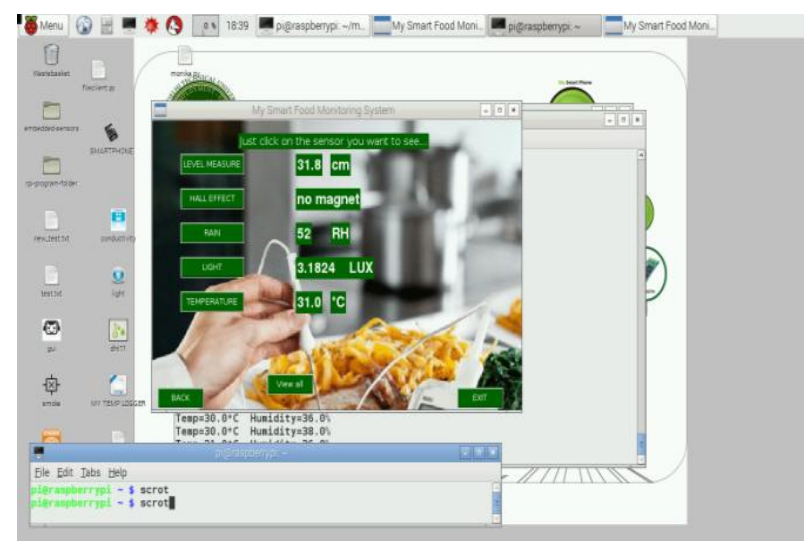

Fig3.GUI showing real time sensor values
The processed sensor values needs to be transferred either via Bluetooth or WiFi. A facility of data logging is also provided. Whenever the user taps the Send alerts button on Page One, the control gets navigated to another frame which comprises of three buttons namely "Create Log", "Bluetooth" and "Internet".

If the user selects the Create Log button, a frame comprising of different sensors labels appears and the user needs to choose the desired sensor for respective log generation.

If Bluetooth button is selected by the user, a frame that comprises of different sensors labels appears. Upon user selection, a particular log is sent over to the android application via Bluetooth. Similarly, there is an internet button option for data transmission over WiFi(Fig 4.).

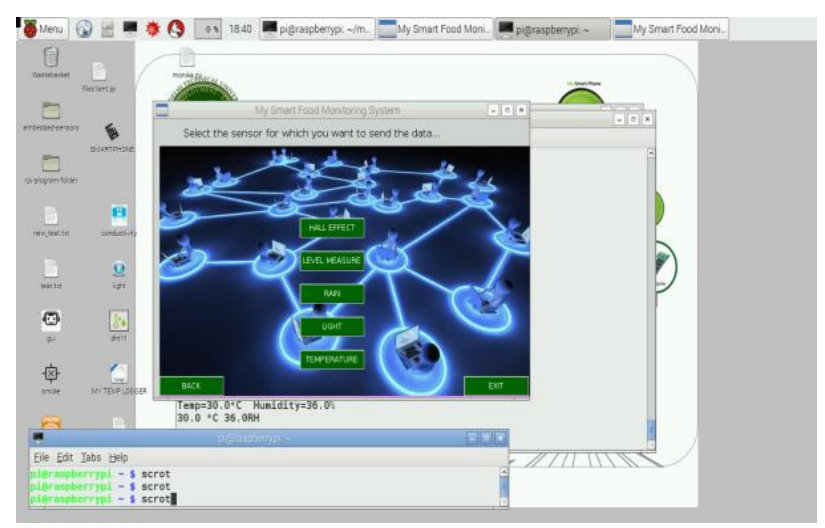

Fig4.Sensor data being transmitted over Wi-Fi

For prototype implementation, a touchscreen is interfaced with raspberry pi to mimic the desktop GUI as shown in Fig.5.

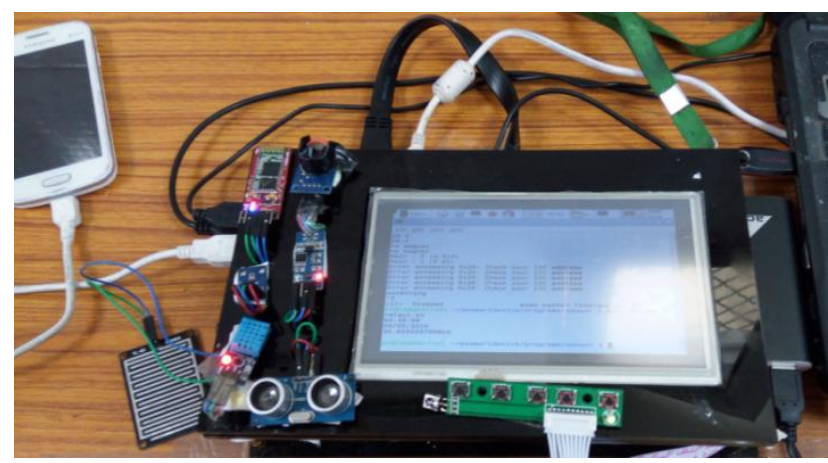

Fig5.Sensor data being transmitted over Wi-Fi

\section{FUTURE WORK}

An app needs to be designed which cannot only communicate the messages to the user but will also be capable of processing the raw data values received from the sensors. Conveying alert messages in the form of emoticons to convey the various degrees of damage to the food so that the messages can be understood by a variety of users still needs to be investigated.

\section{ACKNOWLEDGMENTS}

We would like to thank Design And innovation Centre MHRD for supporting us through the course of this project.

\section{REFERENCES}

[1] Ki-hwan Eom, Chang Won Lee, Nghia Truong Van, Kyung Kwon Jung, Joo Woong Kim And Woo Seung Choi "Food Poisoning Prevention Monitoring System Based On The Smart RFID Tag System" InInternational 
Journal Of Multimedia And Ubiquitous Engineering Vol.8, No.5 (2013), Pp.213-222.

[2] Kong Xiangsheng, "Design And Implementation Of Food Monitoring System Based On Wsn", Xinxiang University, Xinxiang, China.

[3] Karleigh Huff "Active And Intelligent Packaging: Innovations For The Future" Graduate Student Department Of Food Science And Technology Virginia Polytechnic Institute And State University (Virginia Tech) Blacksburg.

[4] "Food Borne Patogen Detection" In Adv. J. Food Sci. Technol., 6(11): 1241-1248, 2014.

[5] Evangelyn C. Alocilja, Member, IEEE, Nichole L. Ritchie, And Daniel L. Grooms " Protocol Development Using Awn Electronic Nose For Differentiating E. Coli Strains" IEEE SENSORS JOURNAL, VOL. 3, No. 6, December 2003

[6] Kang Along, Zhang Chenrui, Zongwei Luo, Lai Xiaozheng, Han Tao "SAWRFID Enabled Multifunctional Sensors For Food Safety Applications" Program For The IEEE International Conference On Rfidtechnology And Applications, 17 - 19 June 2010 Guangzhou, China.

[7] P.-Y. Cresson , C. Ricardi , L. Dubois, S. Vaucher, T. Lasri , J. Pribetich «Temperature Measurement By Microwave Radiometry" I2MTC 2008 - IEEE International Instrumentation And Measurement Technology Conference Victoria, Vancouver Island, Canada, May 12-15, 2008

[8] Ove Schimmer1, Frank Daschner2 And Reinhard Knöchel „Uwb-sensors In Food Quality Management - The Way From The Concept To Market" PROCEEDINGS OF THE 2008 IEEE INTERNATIONAL CONFERENCE ON ULTRA-WIDEBAND (ICUWB2008), VOL. 2

[9] Zhibo Pang, Jun Chen, Zhi Zhang, Qiang Chen, Lirong xuZheng "Global Fresh Food Tracking Service Enabled By Wide Area Wireless Sensor Network “

[10] Fojan P, Jensen KR And Gurevich “ Label-free Detection Of Biomolecular Interaction - DNA - Antimicrobial Peptide Binding" IEEE 2011.

[11] Dr. Jiten Ch. Dutta "Ion Sensitive Field Effect Transistor For Applications In Bioelectronic Sensors: A Research Review" Cisp2012|proceedings|185

[12] Lei Wan1, Yajie Qin1, Patrick Chiang1, 2, Guoping Chen1, Ran Liu1, Zhiliang Hong1 "High-Sensitivity Photodetection Sensor Front-End, Detecting Organophosphourous Compounds for Food Safety"[13] Huy Quoc Nguyen, Bao Quoc Ta, Nils Hoivik, Einar Halvorsen, and Knut E. Aasmundtveit " Carbon Nanotube Based Gas Sensor for Expiration Detection of Perishable Food" Proceedings of the 13th IEEE International
Conference on Nanotechnology Beijing, China, August 58,2013

[13] Dr.M.S.Saravanan, Jeyanta Kumar Singh, N.Thirumoorthy "RFID Sensors for Food Safety Centre by Identifying the Physical Factors that Affecting the Food".

[14] Alok Shah* and OP Chauhan, "Applications of Biosensors in Agri-food industry and mitigation of bio-threat paradigm" Proceedings of the 2015 2nd International Symposium on Physics and Technology of Sensors, 810th March, 2015, Pune, India

[15] Sarita B. Dhoble, N. K. Choudhari, A. R. Choudhari "Electronic Sensor Based System for Analysis of Infection Behavior in Milk" 2015 International Conference on Pervasive Computing (ICPC)

[16] Kwag Dong Kyeung, Research for the presentation of food borne illness food service group, Food and Drug Administration Research Report, 2002.

[17] Raspberry Pi, "Raspberry Pi basics".[online].Available: https://www.raspberrypi.org/products/raspberry-pi-2model-b/.

[18] Seed Studio, "Difference between raspberry pi and intel galileogen2".[online].Available:http://www.seeedstudio.c om/blog/2014/10/31/linkit-one-vs-edison-vs-raspberry-pivs-beaglebone-black/.

[19] Micropik, "Temprature sensor DHT11 interfacing". [online].Available:http://www.micropik.com/PDF/dht11.p df.

[20] Adafruit."Light sensor basics"[online].Available https://cdn-shop.adafruit.com/datasheets/TSL2561.pdf.

[21] Texas Instruments."Hall effect sensor basics".[online].Available:http://www.ti.com/lit/ds/symlin k/drv5023.pdf.

[22] SensorWiki."Ultrasonic Sensor SR 04".[online].Available:http://sensorwiki.org/doku.php/sen sors/ultrasound.

[23] MQ3."Air quality sensor MQ3 basics and interfacing diagrams".[online].Available:https://www.sparkfun.com/d atasheets/Sensors/MQ-3.pdf.

[24] Adafruit, "WiFI Module".[online].Available: https://www.adafruit.com/product/814.

[25] Food Safety Surveillance and Monitoring System in Korea Ki Hwan Park, PhD President, ILSI Korea Professor, Dep. of Food Sci. \& Tech.,Chung-Ang University, Korea Presented under MFDS permission.

[26] ScienceDirect, "Food choices health and environment".[online].Available www.scienceDirect.com/food/1231. 